
DR ARNAUD GREGOIRE L'HUILLIER (Orcid ID : 0000-0001-9230-7285)

MR. TERRANCE KU (Orcid ID : 0000-0002-6757-3757)

DR DEEPALI KUMAR (Orcid ID : 0000-0003-1961-0477)

Article type : Brief Communication

\section{Safety and Immunogenicity of Adjuvanted Recombinant Subunit Herpes Zoster Vaccine in}

\section{Lung Transplant Recipients}

Cedric Hirzel ${ }^{1,2}$, Arnaud G. L'Huillier ${ }^{1,3}$, Victor H. Ferreira ${ }^{1}$, Tina Marinelli ${ }^{1}$, Terrance $\mathrm{Ku}^{1}$, Matthew Ierullo ${ }^{1}$, Congrong Miao ${ }^{4}$, D. Scott Schmid ${ }^{4}$, Stephen Juvet ${ }^{5}$, Atul Humar ${ }^{1}$, and Deepali Kumar $^{1}$

1) Transplant Infectious Diseases and Ajmera Transplant Centre, University Health Network, Toronto, Ontario, Canada

2) Department of Infectious Diseases, Bern University Hospital, University of Bern, Bern, Switzerland

3) Pediatric Infectious Diseases Unit, Department of Child and Adolescent Medicine, Geneva University Hospitals and Medical School, Geneva, Switzerland

4) Division of Viral Diseases, Centers for Disease Control and Prevention, Atlanta, Georgia.

5) Toronto Lung Transplant Program, University Health Network, Toronto, Ontario, Canada

Cedric Hirzel and Arnaud G. L'Huillier contributed equally.

Atul Humar and Deepali Kumar1 share joint senior authorship.

This article has been accepted for publication and undergone full peer review but has not been through the copyediting, typesetting, pagination and proofreading process, which may lead to differences between this version and the Version of Record. Please cite this article as doi: $\underline{10.1111 / \text { AJT.16534 }}$

This article is protected by copyright. All rights reserved 


\section{Correspondence}

Deepali Kumar

Email: deepali.kumar@uhn.ca

\section{Abbreviations}

AI, avidity index

CDC, Center for Disease Control and Prevention

CD40L, CD40 ligand

CMI, cell mediated immunity

CMV, cytomegalovirus

DEA, diethylamine

ELISA, enzyme-linked immunosorbent assay

gE, glycoprotein E

GSK, GlaxoSmithKline

HIV, human immunodeficiency virus

HZ, Herpes zoster

IFN- $\gamma$, interferon- $\gamma$

IL-2, interleukin-2

IgG, Immunoglobulin G

IVIG, intravenous immunoglobulins

IQR, interquartile range

ISHLT, international society for heart and lung transplantation 
OD, optical density

PBS, phosphate buffered saline

PBMC, peripheral blood mononuclear cell

$\mathbf{R Z V}$, recombinant herpes zoster vaccine

SFC, spot forming cell

SOT, solid organ transplant

TNF- $\boldsymbol{\alpha}$, tumor necrosis factor $-\alpha$

SPICE, simplified presentation of incredibly complex evaluations

U.S., United States

$\mathbf{V Z V}$, varicella zoster virus 


\section{ABSTRACT}

Lung transplant recipients are at high risk for herpes zoster and preventive measures are a significant unmet need. We investigated the safety and immunogenicity of two doses of a recombinant zoster vaccine (RZV) in lung transplant recipients ( $\geq 50$ years). We enrolled 50 patients of which 49 received at least one vaccine dose. Anti-glycoprotein E (gE) antibody levels $(n=43)$ increased significantly compared to baseline (median optical density [OD] 1.96; interquartile range [IQR]: 1.17-2.89) after the first (median OD 3.41, IQR 2.54-3.81, $<<0.0001$ ) and second vaccine dose (median OD 3.63, IQR 3.39-3.86, p<0.0001). gE-specific polyfunctional CD4+ T-cell frequencies $(n=38)$ also increased from baseline (median 85 per $10^{6}$ CD4+ T-cells; IQR: 46-180) to the first (median 128 per $10^{6}$ CD4+ T-cells; IQR: 82-353; $\mathrm{p}=0.023$ ) and after the second dose (median 361 per $10^{6}$ CD4+ T-cells; IQR: 146-848; $\left.<<0.0001\right)$. Tenderness $(83.0 \%$; 95\%CI:69.2-92.4\%) and redness (31.9\%; 95\%CI:19.1-47.1\%) at injection site were common. One rejection episode within three weeks of vaccination was observed. This is the first study demonstrating that RZV was safe and elicited significant humoral and cell mediated immunity in lung transplant recipients. RZV is a new option for the prevention of shingles in this population.

\section{INTRODUCTION}

Varicella zoster virus (VZV) infects humans early in life and thereafter establishes lifelong latency in sensory nerve ganglia. ${ }^{1}$. Herpes zoster $(\mathrm{HZ})$ results from reactivation of latent VZV, often causing painful dermatomal eruption or disseminated disease. Complications of VZV occur in 15$40 \%$ of cases, the most common being post-herpetic neuralgia ${ }^{2} \cdot 3,4$. Individuals with diminished 
cellular immunity to VZV are at increased risk for $\mathrm{HZ}$ and the rate of complications is significantly higher in this population ${ }^{5,6}$. Notably, solid organ transplant (SOT) recipients have a predominant cellular immune deficit, caused by chronic immunosuppressive treatment after transplantation. As such, the HZ incidence rate is significantly higher in SOT recipients compared to the general population ${ }^{7,8}$. However, the $\mathrm{HZ}$ risk varies depending on the type of transplanted organ: lung transplant recipients in particular are more likely to reactivate VZV compared to abdominal organ transplant recipients ${ }^{7,8}$. Epidemiologic studies report a cumulative HZ incidence in lung transplant recipients of $15-20 \%$ within the first five years after transplantation 7,9 . Recurrent HZ is common (13.8\%) in lung transplant recipients and a high proportion of patients $(20 \%)$ develop post-herpetic neuralgia ${ }^{9}$.

The adjuvanted recombinant zoster vaccine (RZV; Shingrix ${ }^{\mathrm{TM}}$, GSK) is a two-dose non-live vaccine that contains $50 \mu \mathrm{g}$ of the external domain of the VZV glycoprotein $\mathrm{E}$ (gE) in combination with the AS01B Adjuvant System. RZV is efficacious in preventing HZ in adults $\geq 50$ years of age and is licensed in North America and Europe for this indication ${ }^{10,11}$. A recent study also showed that RZV was immunogenic and safe in kidney transplant recipients aged $\geq 18$ years, under chronic immunosuppressive therapy ${ }^{12}$.

There is an urgent need for HZ preventive strategies in high-risk patients such as lung transplant recipients. However, due to the intense immunosuppressive therapy required to avoid allograft rejection in this population compared to other types of organ transplants, the ability to mount an immune response to vaccines may be limited. We therefore assessed the immunogenicity and safety of RZV in lung transplant recipients in this study.

\section{MATERIALS AND METHODS}

\subsection{Study Design and Participants}

This single arm RZV immunogenicity study was conducted as part of a tertiary care lung transplant program at the University Health Network Transplant Centre, Toronto. Consecutive single or double lung transplant recipients that were $\geq 50$ years of age were approached in outpatient transplant clinic from March 2018 to August 2018. Patients were eligible if they were $\geq$ 90 days post-transplant and had positive varicella zoster IgG antibodies before transplantation (see 
details in Supporting Information). The study was registered at ClinicalTrials.gov (NCT03493776).

After obtaining written informed consent, lung transplant recipients received two intramuscular doses of RZV $(0.5 \mathrm{~mL})$ two to six months apart. Serum and whole blood were obtained immediately before the first vaccination (baseline), immediately before the second vaccination (T1) and three to six weeks after the second vaccination (T2). The primary outcomes of the study were cellular and humoral immune responses to RZV vaccination. The secondary outcome was the safety of the vaccine. 


\subsection{Assessment of Immunogenicity}

The humoral immune response was evaluated using enzyme-linked immunosorbent assay (ELISA) targeting VZV glycoprotein E (gE) antibodies at T1 and T2 as compared to baseline ${ }^{13}$. Testing was performed at the VZV reference laboratory at the Centers for Disease Control and Prevention (CDC, Atlanta, USA). Results were expressed as optical density (OD) values. Avidity testing of anti-gE antibodies was also performed.

In order to assess the cell mediated immune response we modified previously described methods 14,15. The frequencies of gE-specific $\mathrm{CD}^{2+}{ }^{+} \mathrm{T}$-cells $(\mathrm{CD} 4+\mathrm{T}$-cells expressing two or more activation markers of the four assessed: interferon- $\gamma$, interleukin- 2 , tumor necrosis factor- $\alpha$, and CD40 ligand) were measured, after in vitro stimulation with a pool of 15315 -mer peptides (1.25 $\mu \mathrm{g} / \mathrm{mL}$ each, overlapping by 11 amino acids) which span the entire VZV gE protein (JPT Peptide Technologies, Berlin, Germany), by intracellular cytokine staining and detection by flow cytometry as previously established by others ${ }^{12,16-22}$. Details are provided in the Supporting Information.

\subsection{Assessment of Reactogenicity and Safety}

Standardized diaries were provided to all participants to record solicited local and general adverse events for 7 days after each vaccination. ( see details in Supporting Information). All solicited local and generalized symptoms were considered causally related to the vaccine. Unsolicited adverse events such as hospitalizations and rejection episodes were collected until three months after the last vaccine dose. For patients who had only one vaccine dose, unsolicited adverse events were collected until the latest possible time point for administration of the second dose (6 months after the first vaccine). Allograft rejection was defined as biopsy proven or clinically suspected and treated. We also performed a 2-year follow-up by chart review of these patients for the occurrence of shingles.

\subsection{Statistical Analysis}

The sample size of 50 patients was based on feasibility and enrollment of at least $10 \%$ of the prevalent lung transplant population over age 50 years during the study period. Reactogenicity and safety results were analyzed in all participants who were vaccinated with at least one dose of RZV. The analyses of humoral and cellular immune responses were performed in patients for whom all 
baseline and post-vaccine specimens were available (provided the PBMC counts allowed the assay to be performed) (Figure 1). For group comparisons, Chi-squared or Fisher exact test were used for dichotomous variables, whereas Mann-Whitney U test was used for continuous variables. We used Wilcoxon signed rank test for paired analysis. p-values $<0.05$ were considered statistically significant. Statistics were done in GraphPad version 7.0 (La Jolla, CA, USA) and in STATA version 16.0 (Stata Corp., College Station, TX). Graphs were made using GraphPad version 7.0 (La Jolla, CA, USA) or National Institute of Health (NIH)'s Simplified presentation of incredibly complex evaluations (SPICE) ${ }^{23}$.

\section{RESULTS}

\subsection{Study Participants}

We enrolled a total of 50 lung transplant recipients. Of these, 49 patients received at least one vaccine dose (Figure 1). The median age of participants was 65.4 years (IQR: 59.1-70.6). Most participants were men $(29 / 49 ; 59.2 \%)$ and most had a double vs. single lung transplant $(40 / 49$; $80.1 \%$ ) (Table 1). The median time from transplant to the first vaccination was 3.0 years (IQR: 1.0-5.0). The median interval between the first and second vaccine was 63 days (IQR: 62-69). Except for one patient, all participants were on triple immunosuppressive treatment with median trough levels for cyclosporine (192 ng/mL; IQR: 155-227) or tacrolimus (12.5 ng/mL; IQR: 7.214.9) at time of the first vaccine.

\subsection{Humoral Immune Response}

Serum samples at all time points were available for 43 lung transplant recipients (Figure 1). The VZV gE antibody concentration increased significantly compared to baseline (median OD 1.96; IQR: 1.17-2.89) after the first (median OD 3.41, IQR 2.54-3.81, $<<0.0001$ ) and the second vaccine dose (median OD 3.63, IQR 3.39-3.86, p<0.0001; Figure 2A). At baseline, gE-specific antibody avidity was relatively low (46.4\%, IQR: 36.6-63.4). Noteworthy, the avidity of the antibodies increased significantly after the first (71.5\%; IQR: 52.9-91.5; $\mathrm{p}<0.0001)$ and the second vaccine (84.2\%; IQR: 59.4-96.5; $\mathrm{p}<0.0001$ ) (Figure 2B). There was a strong positive correlation between VZV anti-gE antibody concentrations (as by OD) and the avidity of anti-gE antibodies at baseline (Spearman's rho $=0.6442 ; \mathrm{p}<0.0001$ ) after the first (Spearman's rho $=0.8325 ; \mathrm{p}<0.0001$ ) and after 
the second vaccine (Spearman's rho=0.7338; $\mathrm{p}<0.0001$ ). Further analysis did not show a significant correlation between anti-gE antibody concentrations and patient age or time from transplant to vaccination (data not shown) but showed an inverse correlation between dose of mycophenolate and antibody concentration (Spearman's rho= $-0.3447 ; \mathrm{p}=0.019$ ).

\subsection{Cell Mediated Immune Response}

Cell mediated immune responses were analyzed for 38 patients (Figure 1). VZV gE-specific $\mathrm{CD}^{2+}{ }^{2+} \mathrm{T}$-cell frequencies increased significantly from baseline (median $85 \mathrm{CD}^{2+}{ }^{\mathrm{T}}$-cells per $10^{6}$ CD4 T-cells; IQR: 46-180) to after the first vaccine (median $128 \mathrm{CD}^{2+}{ }^{+} \mathrm{T}$-cells per $10^{6} \mathrm{CD} 4 \mathrm{~T}$ cells; IQR 82-353; $\mathrm{p}=0.023$ ) and after the second vaccine (median $361 \mathrm{CD} 4{ }^{2+} \mathrm{T}$-cells per $10^{6} \mathrm{CD} 4$ T-cells; IQR: 146-848; p<0.0001) (Figure 3). Among VZV gE-specific CD4 ${ }^{2+}$ T-cells, IL2+CD40L + CD4 T-cells contributed the most to the polyfunctional signature (Figure 4). There was no significant increase in VZV-specific CD8 T-cells due to vaccination (data not shown).

Patients with a longer interval between transplantation and vaccination had higher frequencies of $\mathrm{CD}^{2+}{ }^{+} \mathrm{T}$-cells at baseline (Spearman's rho $=0.4118 ; \mathrm{p}=0.0102$ ) and a trend towards higher $\mathrm{gE}$ specific $\mathrm{CD}^{2+}{ }^{2} \mathrm{~T}$-cells after the second vaccine dose (Spearman's rho $=0.3184 ; \mathrm{p}=0.0514$ ). There was no correlation with age and the frequencies of $\mathrm{CD}^{2+} \mathrm{T}$-cells at baseline or after vaccination (data not shown).

Anti-gE antibody concentrations (as by OD) and the number of gE-specific CD4 ${ }^{2+} \mathrm{T}$-cells were not correlated at any of the different time points (data not shown).

\subsection{Reactogenicity and Safety}

Forty-nine participants received at least one vaccine dose (included in the safety analysis for unsolicited adverse events). Of these, $47 / 49$ completed the standardized diary for capturing solicited adverse events after the first vaccine dose and 44/45 completed the diary after the second vaccine dose.

During the seven day post-vaccination follow-up for solicited adverse events, the most common local adverse event was tenderness at injection site reported by $83.0 \%$ (95\% CI: $69.2-92.4 \%$ ) (39/47), followed by redness (31.9\%; 95\% CI: 19.1-47.1\%) (15/47) and swelling (26.7\%; 95\% CI: $14.6-41.9 \%)(12 / 45)$. Only a minority of these events were classified as grade 3 ; all were selflimited (Figure 5). When occurring, local adverse events were of short duration. Tenderness at 
injection time resolved after a median of 2 days (IQR: 2-3 days), so did redness (IQR: 1-3 days), and swelling (IQR: 2-3 days). The most frequent generalized solicited symptoms were myalgia (36.2\%; 95\% CI 22.7-51.5; 17/47), fatigue (31.9\%; 95\% CI 19.1-47.1; 15/47), and headache (23.4\%; 95\% CI 12.3-38.0; 11/47). Gastrointestinal complaints $(6.4 \%$; 95\% CI 1.3-17.5; 3/47) and shivering $(4.3 \%$; $95 \%$ CI $0.5-14.5 ; 2 / 47)$ were rare and no participant developed fever within seven days after injection.

From the first vaccination to months 3 after the last immunization we recorded 26 unsolicited adverse events of which 14 were severe adverse events (Table E1 Supporting Information). Throughout the study, one death was reported (respiratory failure due to laboratory-confirmed influenza B infection) which we considered unrelated to vaccination. Two patients developed a $\mathrm{HZ}$ infection during the study 2-year follow-up for occurrence of shingles (with no cases occurring after the second vaccine dose): one participant experienced a mono-dermatomal infection two days after the first vaccine dose. The patient had treatment with valacyclovir and recovered without sequelae. In retrospect, this patient already had symptoms of reactivation (pain at reactivation site) at the time of vaccination. The second participant developed a disseminated herpes zoster episode 32 days after the first vaccine dose. This patient's lesions resolved with intravenous acyclovir although he developed post-herpetic neuralgia.

Three participants, all of whom were within the first year post-transplant, experienced four rejection episodes (none documented to be antibody-mediated). One lung transplant recipient had two episodes of clinically suspected rejection 89 and 130 days after the first vaccination (both episodes before the second vaccine dose), respectively. Due to the long interval between suspected rejection and RZV immunization these rejection episodes were classified as unrelated to vaccination. Two participants experienced a histologically proven cellular rejection episode (both grade A1 according to the ISHLT classification ${ }^{24}$ ) at 17 days and 90 days after second vaccination respectively.

Protocol biopsies and DSA measurements for clinical purposes in our program were done at $1,3,6,9,12,18,24$ months post-transplant and repeated if rejection was suspected. Based on this, there were eleven patients in the study cohort (all recently transplanted within the first 2 years) who had DSA measured in the 3 months prior to first vaccine and up to 3 months after the second dose of vaccine. Of these, only one patient developed new weak positive DSA compared to pre- 
vaccination. The patient was 9 months post-transplant and a biopsy done at that time showed no rejection.

\section{DISCUSSION}

We conducted a study to assess the immunogenicity and safety of recombinant zoster vaccine in lung transplant recipients $\geq 50$ years of age. The major findings of our study were as follows: (1) We found that RZV vaccination elicits a significant increase in VZV gE specific antibody; (2) the vaccine also enhances the strength (avidity) with which IgG antibodies bind to VZV gE; (3) RZV induces a significant cell mediated immune response as determined by the number of VZV gEspecific polyfunctional CD4 T-cells; (4) adverse events at the injection site were common but of short duration and of mild severity; and (5) there were no severe rejection episodes with temporal association to the vaccine; and (6) no clinical cases of zoster were observed after the second vaccine dose during a two-year follow-up period.

To our knowledge, this represents the first study that systematically examines serologic and cellular immune responses to RZV in lung transplant recipients, the most vulnerable group to HZ among SOT recipients ${ }^{7}$. Our findings are consistent with previously reported studies showing that RZV elicits anti-gE humoral and cellular immune responses in non-immunocompromised individuals $\geq 50$ years of age ${ }^{19-21,25,26}$.

The only study published so far that examined RZV-associated immune responses in organ transplant recipients, was restricted to kidney transplant patients ${ }^{12}$. The humoral vaccine response rate (defined as $\geq 4$-fold increase in antibody titer) was $80.2 \%$ and no apparent difference in anti$\mathrm{gE}$ antibody geometric mean antibody concentration was seen linked to the types of maintenance immunosuppressive therapy. Humoral immune responses appeared lower for participants $\geq 50$ years of age compared to younger kidney transplant recipients. Median gE-specific CD4 ${ }^{2+}$ frequencies pre-vaccination were low $\left(21\right.$ cells $/ 10^{6} \mathrm{CD} 4 \mathrm{~T}$-cells $)$ and increased significantly four weeks after the second vaccine dose $\left(2149\right.$ cells $/ 10^{6} \mathrm{CD} 4 \mathrm{~T}$-cells). Consistent with the findings in kidney transplant recipients, we also found a significant antibody response in lung transplant recipients after RZV immunization. Since our antibody assay was a CDC performed assay using optical density vs. the in-house GSK assay used in other studies, an exact comparison of antibody 
concentrations between studies is not possible. However, this is of less relevance because the antibody cut-offs for $\mathrm{HZ}$ prevention are not known. Nonetheless, we do demonstrate that there is a statistically significant increase in antibody levels after vaccination in lung transplant recipients $(p<0.001)$. In addition, a unique aspect of our assessment including an antibody avidity assessment which provides further evidence of a robust and meaningful antibody response postvaccination. In accordance with the previously reported results for non-immunocompromised individuals ${ }^{19-21,26}$ and for kidney transplant patients ${ }^{12}$ we also found a significant increase in polyfunctional gE-specific T-cells.

In the immunocompetent population $\mathrm{IL}-2^{+} \mathrm{CD} 40 \mathrm{~L}^{+}$double-positive cells account for the majority of vaccine induced $\mathrm{gE}$-specific $\mathrm{CD} 4^{2+} \mathrm{T}$-cells ${ }^{20,21}$. Consistent with the findings in immunocompetent individuals, the largest $\mathrm{gE}$-specific $\mathrm{CD} 4{ }^{2+}$ cell population after RZV immunization of lung transplant recipients were also IL-2+CD40L+ double-positive cells. Therefore, the immunosuppressive treatment associated with lung transplantation does not obviously affect the signature of polyfunctional gE-specific CD4 T-cells.

There was a weak to moderate, statistically significant, inverse correlation (Spearman's rho= 0.3447; $\mathrm{p}=0.019$ ) of mycophenolate dose and vaccine elicited antibody concentration. An inverse correlation of mycophenolate dose and vaccine-elicited antibody response is an observed phenomenon that has previously been noted in studies of influenza vaccines in the transplant setting ${ }^{27}$. However, pre-emptive reduction in mycophenolate doses has not been studied and is generally not advised to overcome the reduced immune response.

Reactions at the injection site such as tenderness (83.0\%), redness (31.9\%) and swelling (26.7\%) were common after immunization. The frequency of these reactions among lung transplant patients is similar compared to kidney transplant recipients ${ }^{12}$ and non-immunocompromised individuals 10,11,19,21. We recorded two biopsy proven rejection episodes and two clinically suspected and treated rejection episodes after RZV vaccination. All rejection episodes were mild and three out of four rejection episodes occurred more than 30 days after the last vaccine dose and were therefore considered unrelated to RZV vaccination. All episodes were in patients that were within the first post-transplant year. According to ISHLT registry data, $26.6 \%$ of lung transplant recipients will require treatment for acute rejection in the first year post-transplant ${ }^{28}$. The frequency of biopsy proven allograft rejections in kidney transplant recipients was similar in the 
placebo group (3.0\%) compared to RZV vaccination (5.3\%) in a previous study ${ }^{12}$. In our study, two patients developed HZ after the first vaccine. The first patient already had symptoms at enrollment and the second patient developed the infection $>30$ days after the first dose of vaccine. We therefore did not consider these events related to the vaccine.

One strength of our study includes its unique population of highly immune suppressed lung transplant recipients and an in-depth analysis of the vaccine elicited immune response, including quantitative and qualitative antibody responses and cell mediated immune response. By choosing the polyfunctional CD4+ T-cell response as a primary endpoint for cell-mediated immunity we ensured that we are assessing CD4 responses of high quality, capable of eliciting potent and durable T-cell responses ${ }^{29}$. This approach has previously been chosen by others to characterize gE-specific CMI ${ }^{12,16-22}$ and it has been shown that CD4+ T-cells which express at least two activation markers are more long-lasting compared to single positive cells ${ }^{20}$. A limitation of our study is the absence of a control group although a placebo control would not be ethical since in Canada, vaccine is recommended for everyone over age 50 years. This means that the rate of adverse events after vaccination may be overestimated in our study, as it cannot be compared to adverse events after placebo injection. In addition, the lack of a healthy population comparator means that we are unable to compare relative immunogenicity. However, there are also no serological or CMI correlates of protection established for vaccine efficacy. In addition, since standardized measurements of gE-specific CMI and gE-specific antibodies are not available, the counts of gE-specific polyfunctional CD4+ T-cells and the concentrations of antibodies cannot be directly compared with previous studies using different assays. Although our study population was small, it was sufficient to provide immunologic and safety data and represented $10 \%$ of our total prevalent lung transplant cohort $\geq 50$ years of age. The study did not examine the long-term durability of immune responses or memory B-cell formation. These aspects could be a focus for future studies.

In summary, we provide novel information on the immune response elicited by RZV vaccination in lung transplant recipients. We show that the vaccine induces cell mediated and humoral immune responses in this severely immunocompromised population at high risk for herpes zoster. Our study suggests that RZV can be used to prevent shingles in the lung transplant population. 


\section{ACKNOWLEDGMENTS}

This work was supported through internal funding of the Multi-Organ Transplant Program, University Health Network, Toronto, Ontario, Canada. C.H. was supported by an early Postdoc.Mobility grant from the Swiss National Science Foundation (P2BEP3_175265). A.G.L. was supported by an advanced Postdoc. Mobility grant from the Swiss National Science Foundation (P300PB_171603).

\section{DISCLOSURE}

The authors of this manuscript have conflicts of interest to disclose as described by the American Journal of Transplantation. D.K. has received consulting fees from GSK and clinical trials grant from GSK and Merck. The other authors have no conflicts of interest to disclose. The findings and conclusions in this report are those of the authors and do not necessarily represent the official position of the Centers for Disease Control and Prevention (CDC).

\section{DATA AVAILABILITY STATEMENT}

The data that support the findings of this study are available from the corresponding author, upon reasonable request.

\section{ORCID:}

Cedric Hirzel: 0000-0002-7870-912X

Deepali Kumar: 0000-0003-1961-0477 


\section{FIGURE LEGENDS}

Figure 1 Participant flowchart

Abbreviations: Cell mediated immunity (CMI); post-dose 1 visit (T1); post-dose 2 visit (T2);

Herpes Zoster (HZ); Intravenous Immunoglobulin (IVIG); Varicella Zoster Virus (VZV)

Figure 2 Antibody response to recombinant herpes zoster subunit vaccine

$A$, anti-glycoprotein E antibody concentration expressed as optical density. Whiskers represent maximal and minimal values. Boxes delimit upper and lower quartiles. $B$, anti-glycoprotein $\mathrm{E}$ antibody avidity. Whiskers represent maximal and minimal values. Boxes delineate upper and lower quartiles. Abbreviations: enzyme linked immunosorbent assay (ELISA); glycoprotein E (gE); optical density (OD).

Figure 3 Cell mediated immune response to recombinant herpes zoster subunit vaccine

Glycoprotein E specific CD4 ${ }^{2+}$ frequencies (polyfunctional CD4+ T-cells) per $10^{6}$ CD4+ T-cells. Whiskers represent maximal and minimal values. Boxes delineate upper and lower quartiles. Abbreviations: glycoprotein E (gE).

Figure 4 Frequency of gE-specific CD4 T-cells expressing at least two activation markers

Bold bar indicates median number of cells. Whiskers indicate upper and lower interquartile range. Abbreviations: CD40 ligand (CD40L); glycoprotein E (gE); interleukin 2 (IL2); interferon gamma (IFN); tumor necrosis factor alpha (TNF).

Figure 5 Solicited adverse events

Error bars indicate $95 \%$ confidence intervals. Fever was defined as a body temperature $\geq 38.0^{\circ} \mathrm{C}$.

*No grading of symptom severity for shivering, gastrointestinal symptoms (nausea / vomiting) and 
fever. N, number of participants with at least one vaccine dose administered and completed adverse events diary. Events occurred either after the first dose or second dose.

This article is protected by copyright. All rights reserved 


\section{REFERENCES}

1. Horien C, Grose C. Neurovirulence of varicella and the live attenuated varicella vaccine virus. Seminars in pediatric neurology. 2012;19(3):124-129.

2. Kost RG, Straus SE. Postherpetic neuralgia--pathogenesis, treatment, and prevention. The New England journal of medicine. 1996;335(1):32-42.

3. Harpaz R, Leung JW. The Epidemiology of Herpes Zoster in the United States During the Era of Varicella and Herpes Zoster Vaccines: Changing Patterns Among Older Adults. Clinical infectious diseases : an official publication of the Infectious Diseases Society of America. 2019;69(2):341344.

4. Ragozzino MW, Melton LJ, 3rd, Kurland LT, Chu CP, Perry HO. Population-based study of herpes zoster and its sequelae. Medicine. 1982;61(5):310-316.

5. Gershon AA, Gershon MD, Breuer J, Levin MJ, Oaklander AL, Griffiths PD. Advances in the understanding of the pathogenesis and epidemiology of herpes zoster. Journal of clinical virology : the official publication of the Pan American Society for Clinical Virology. 2010;48 Suppl 1:S2-7.

6. Yawn BP, Itzler RF, Wollan PC, Pellissier JM, Sy LS, Saddier P. Health care utilization and cost burden of herpes zoster in a community population. Mayo Clinic proceedings. 2009;84(9):787-794.

7. Gourishankar S, McDermid JC, Jhangri GS, Preiksaitis JK. Herpes zoster infection following solid organ transplantation: incidence, risk factors and outcomes in the current immunosuppressive era. American journal of transplantation : official journal of the American Society of Transplantation and the American Society of Transplant Surgeons. 2004;4(1):108-115.

8. Pergam SA, Forsberg CW, Boeckh MJ, et al. Herpes zoster incidence in a multicenter cohort of solid organ transplant recipients. Transplant infectious disease : an official journal of the Transplantation Society. 2011;13(1):15-23.

9. Manuel O, Kumar D, Singer LG, Cobos I, Humar A. Incidence and clinical characteristics of herpes zoster after lung transplantation. J Heart Lung Transplant. 2008;27(1):11-16.

10. Lal H, Cunningham AL, Godeaux O, et al. Efficacy of an adjuvanted herpes zoster subunit vaccine in older adults. The New England journal of medicine. 2015;372(22):2087-2096.

11. Cunningham AL, Lal H, Kovac M, et al. Efficacy of the Herpes Zoster Subunit Vaccine in Adults 70 Years of Age or Older. The New England journal of medicine. 2016;375(11):1019-1032.

12. Vink P, Ramon Torrell JM, Sanchez Fructuoso A, et al. Immunogenicity and Safety of the Adjuvanted Recombinant Zoster Vaccine in Chronically Immunosuppressed Adults Following Renal Transplant: A Phase 3, Randomized Clinical Trial. Clinical infectious diseases : an official publication of the Infectious Diseases Society of America. 2020;70(2):181-190.

This article is protected by copyright. All rights reserved 
13. Hammond $\mathrm{O}$, Wang Y, Green T, et al. The optimization and validation of the glycoprotein ELISA assay for quantitative varicella-zoster virus (VZV) antibody detection. Journal of medical virology. 2006;78(12):1679-1687.

14. Chlibek R, Smetana J, Pauksens K, et al. Safety and immunogenicity of three different formulations of an adjuvanted varicella-zoster virus subunit candidate vaccine in older adults: a phase II, randomized, controlled study. Vaccine. 2014;32(15):1745-1753.

15. Moris P, van der Most R, Leroux-Roels I, et al. H5N1 influenza vaccine formulated with AS03 A induces strong cross-reactive and polyfunctional CD4 T-cell responses. Journal of clinical immunology. 2011;31(3):443-454.

16. Vink P, Delgado Mingorance I, Maximiano Alonso C, et al. Immunogenicity and safety of the adjuvanted recombinant zoster vaccine in patients with solid tumors, vaccinated before or during chemotherapy: A randomized trial. Cancer. 2019;125(8):1301-1312.

17. Berkowitz EM, Moyle G, Stellbrink HJ, et al. Safety and immunogenicity of an adjuvanted herpes zoster subunit candidate vaccine in HIV-infected adults: a phase 1/2a randomized, placebocontrolled study. The Journal of infectious diseases. 2015;211(8):1279-1287.

18. Dagnew AF, Ilhan O, Lee WS, et al. Immunogenicity and safety of the adjuvanted recombinant zoster vaccine in adults with haematological malignancies: a phase 3, randomised, clinical trial and post-hoc efficacy analysis. The Lancet infectious diseases. 2019;19(9):988-1000.

19. Chlibek R, Bayas JM, Collins H, et al. Safety and immunogenicity of an AS01-adjuvanted varicella-zoster virus subunit candidate vaccine against herpes zoster in adults $>=50$ years of age. The Journal of infectious diseases. 2013;208(12):1953-1961.

20. Cunningham AL, Heineman TC, Lal H, et al. Immune Responses to a Recombinant Glycoprotein E Herpes Zoster Vaccine in Adults Aged 50 Years or Older. The Journal of infectious diseases. 2018;217(11):1750-1760.

21. Leroux-Roels I, Leroux-Roels G, Clement F, et al. A phase 1/2 clinical trial evaluating safety and immunogenicity of a varicella zoster glycoprotein e subunit vaccine candidate in young and older adults. The Journal of infectious diseases. 2012;206(8):1280-1290.

22. Grupping K, Campora L, Douha M, et al. Immunogenicity and Safety of the HZ/su Adjuvanted Herpes Zoster Subunit Vaccine in Adults Previously Vaccinated With a Live Attenuated Herpes Zoster Vaccine. The Journal of infectious diseases. 2017;216(11):1343-1351.

23. Roederer M, Nozzi JL, Nason MC. SPICE: exploration and analysis of post-cytometric complex multivariate datasets. Cytometry Part A : the journal of the International Society for Analytical Cytology. 2011;79(2):167-174.

This article is protected by copyright. All rights reserved 
24. Stewart S, Fishbein MC, Snell GI, et al. Revision of the 1996 working formulation for the standardization of nomenclature in the diagnosis of lung rejection. J Heart Lung Transplant. 2007;26(12):1229-1242.

25. Chlibek R, Pauksens K, Rombo L, et al. Long-term immunogenicity and safety of an investigational herpes zoster subunit vaccine in older adults. Vaccine. 2016;34(6):863-868.

26. Levin MJ, Kroehl ME, Johnson MJ, et al. Th1 memory differentiates recombinant from live herpes zoster vaccines. The Journal of clinical investigation. 2018;128(10):4429-4440.

27. Hirzel C, Kumar D. Influenza vaccine strategies for solid organ transplant recipients. Current opinion in infectious diseases. 2018;31(4):309-315.

28. Chambers DC, Cherikh WS, Harhay MO, et al. The International Thoracic Organ Transplant Registry of the International Society for Heart and Lung Transplantation: Thirty-sixth adult lung and heart-lung transplantation Report-2019; Focus theme: Donor and recipient size match. $J$ Heart Lung Transplant. 2019;38(10):1042-1055.

29. Seder RA, Darrah PA, Roederer M. T-cell quality in memory and protection: implications for vaccine design. Nature reviews Immunology. 2008;8(4):247-258.

\section{SUPPORTING INFORMATION STATEMENT}

Additional supporting information may be found online in the Supporting Information section at the end of the article. 


\section{TABLES}

Table 1 Characteristics of lung transplant recipients that received at least one dose of recombinant zoster vaccine.

\begin{tabular}{|c|c|}
\hline Characteristics & $\mathrm{N}=49$ \\
\hline Age (years) [median (IQR)] & $65.4(59.1-70.6)$ \\
\hline Male $(\%)$ & $29(59.2)$ \\
\hline $\begin{array}{l}\text { Time from transplant to first vaccine } \\
\text { (years) [median (IQR)] }\end{array}$ & $3.0(1.0-5.0)$ \\
\hline Within first year of transplantation (\%) & $12(24.5)$ \\
\hline Double lung transplant $(\%)$ & $40(81.6)$ \\
\hline Previous live attenuated herpes zoster vaccine ${ }^{a}(\%)$ & $12(24.5)$ \\
\hline Previous herpes zoster episode ${ }^{a}(\%)$ & $7(14.3)$ \\
\hline Underlying lung disease (\%) & \\
\hline Idiopathic pulmonary fibrosis & $21(42.6)$ \\
\hline COPD & $11(22.4)$ \\
\hline Cystic Fibrosis & $2(4.1)$ \\
\hline Other & $15(30.6)$ \\
\hline Antithymocyte globulin 3-6 months prior (\%) & $2(4.1)$ \\
\hline Basiliximab 3-6 months prior (\%) & $3(6.1)$ \\
\hline Immunosuppression & \\
\hline Prednisone $(\%)$ & $49(100.0)$ \\
\hline Prednisone dose (mg/day) [median (IQR)] & $10(5-10)$ \\
\hline Tacrolimus (\%) & $16(33.3)$ \\
\hline Tacrolimus trough level (ng/ml) [median (IQR)] & $12.5(7.2-14.9)$ \\
\hline Cyclosporin (\%) & $33(66.6)$ \\
\hline Cyclosporin trough level (ng/ml) [median (IQR)] & $192(155-227)$ \\
\hline $\begin{array}{l}\text { Mycophenolate mofetil / Mycophenolate sodium } \\
(\%)\end{array}$ & $41(83.7)$ \\
\hline Azathioprine (\%) & $7(14.3)$ \\
\hline
\end{tabular}


Figure 2 Antibody response to recombinant herpes zoster subunit vaccine

$A$, anti-glycoprotein $\mathrm{E}$ antibody concentration expressed as optical density. Whiskers represent maximal and minimal values. Boxes delimit upper and lower quartiles. $B$, anti-glycoprotein $\mathrm{E}$ antibody avidity. Whiskers represent maximal and minimal values. Boxes delineate upper and lower quartiles. Abbreviations: enzyme linked immunosorbent assay (ELISA); glycoprotein E (gE); optical

A density (OD).

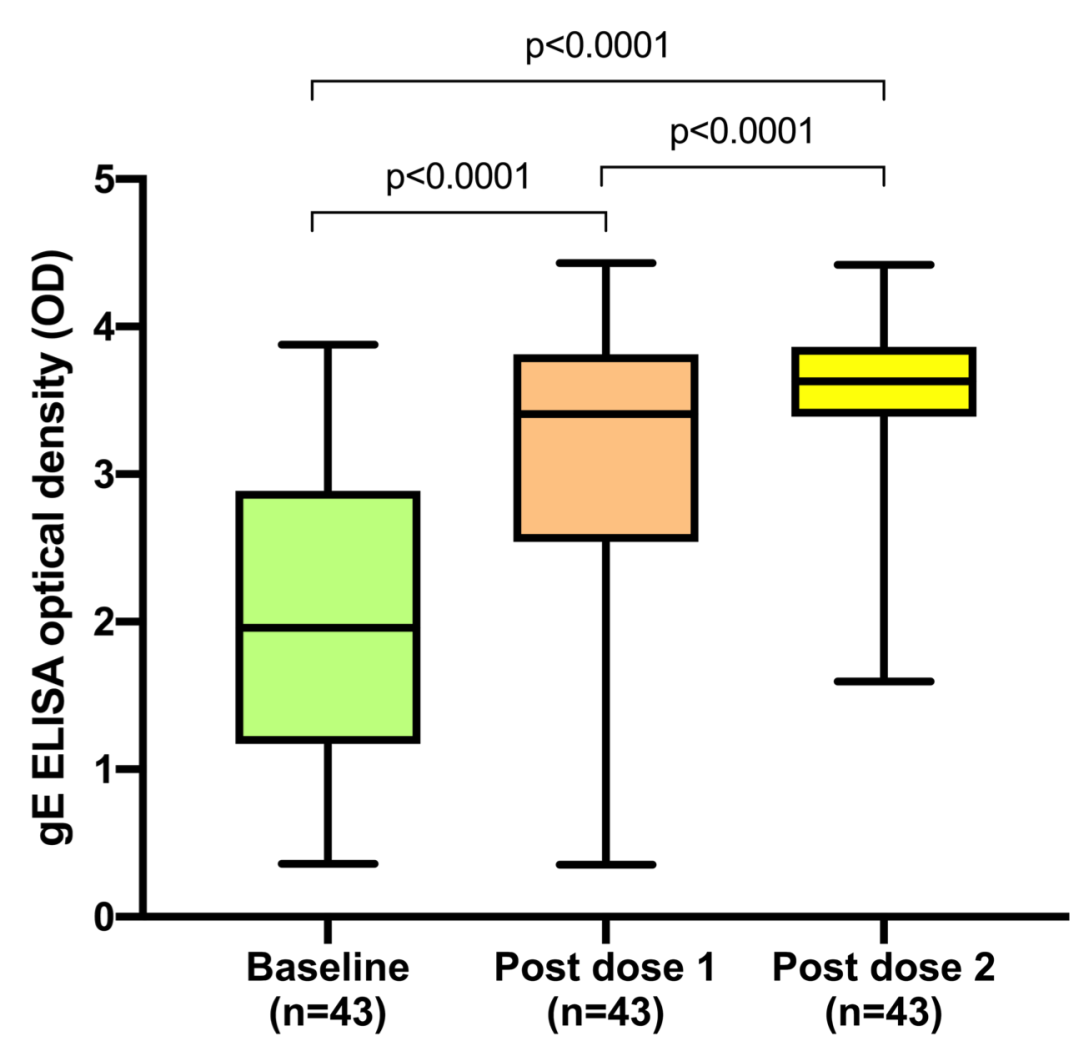

B

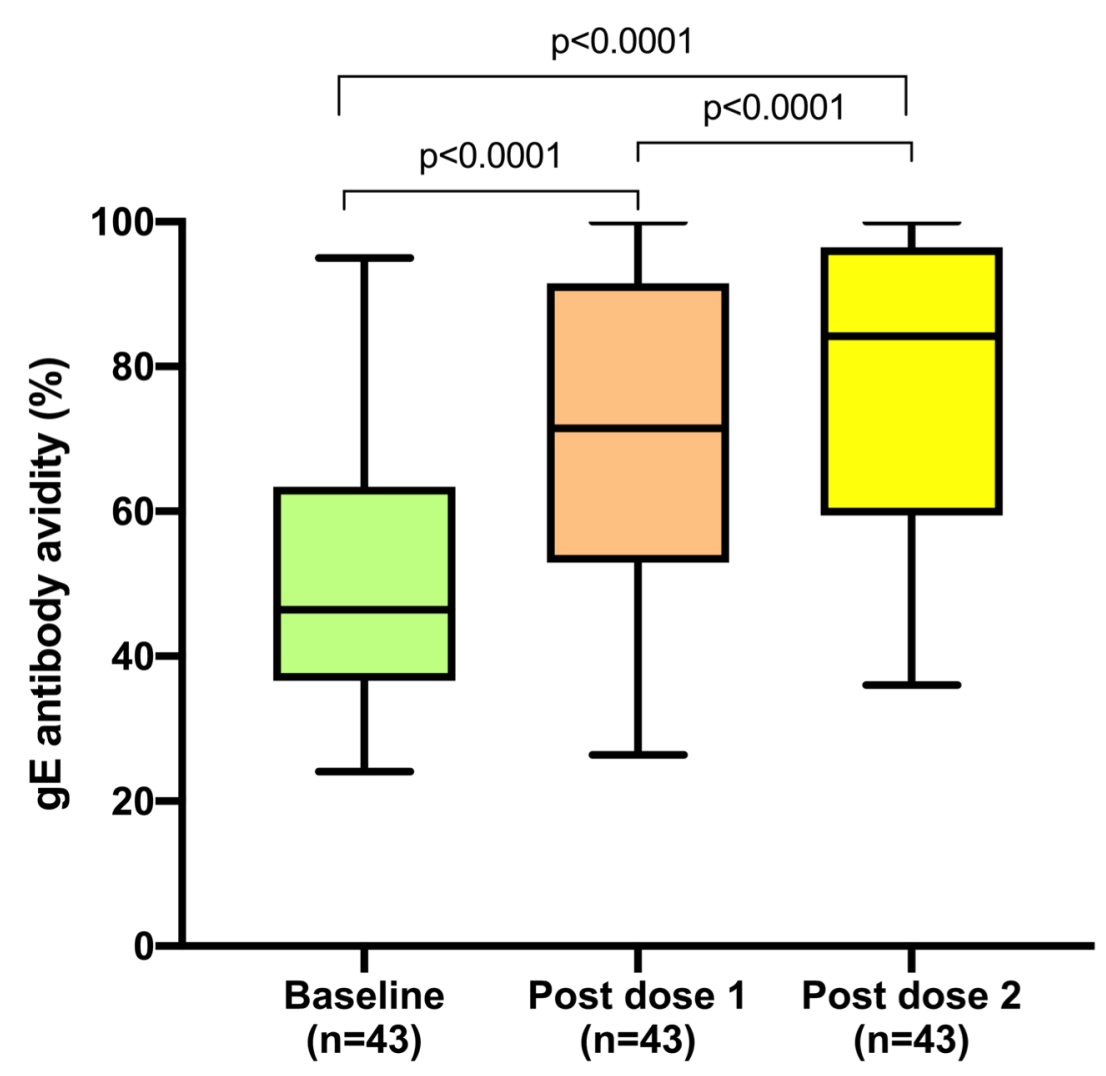

This article is protected by copyright. All rights reserved 
Figure 3 Cell mediated immune response to recombinant herpes zoster subunit vaccine

Glycoprotein E specific CD4 ${ }^{2+}$ frequencies (polyfunctional CD4+ T-cells) per $10^{6} \mathrm{CD} 4+\mathrm{T}-\mathrm{c}$ - lls. Whiskers represent maximal and minimal values. Boxes delineate upper and lower quartiles. Abbreviations: glycoprotein E (gE).

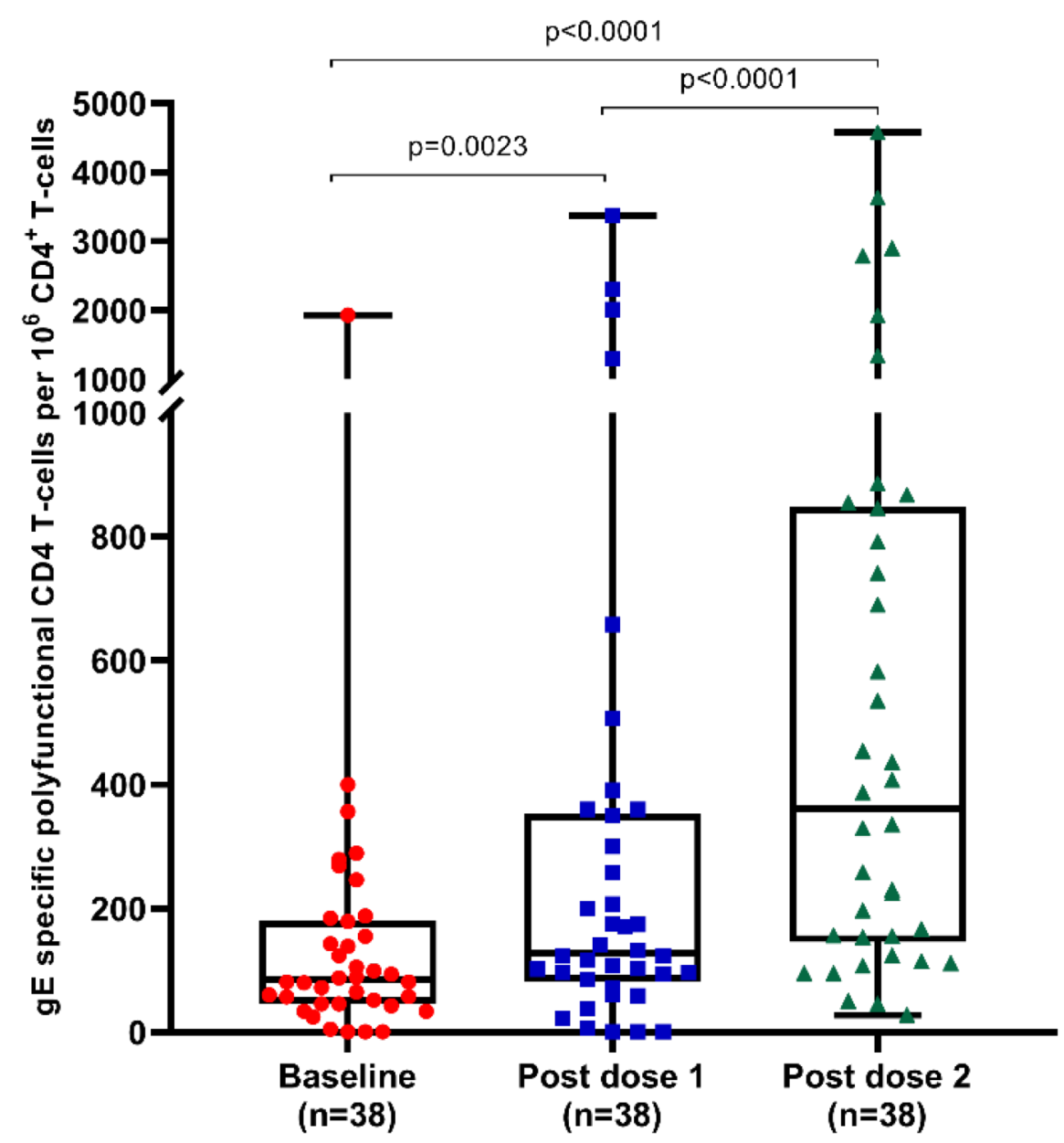

This article is protected by copyright. All rights reserved 
Figure 4 Frequency of gE-specific CD4 T-cells expressing at least two activation markers

Bold bar indicates median number of cells. Whiskers indicate upper and lower interquartile range. Abbreviations: CD40 ligand (CD40L); glycoprotein E (gE); interleukin 2 (IL2); interferon gamma (IFN); tumor necrosis factor alpha (TNF).

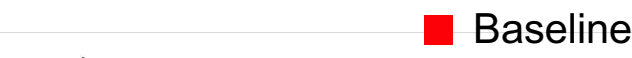

Post dose 1

- Post dose 2

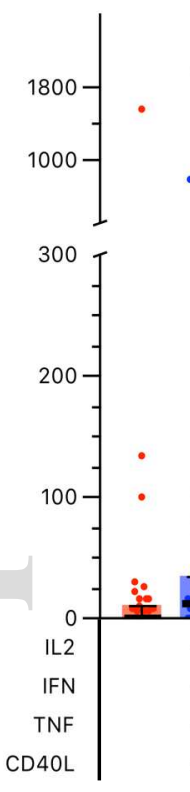

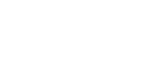

:

$\cdot$

$\vdots$

. $\quad \therefore \quad \therefore$

. $\quad$ :
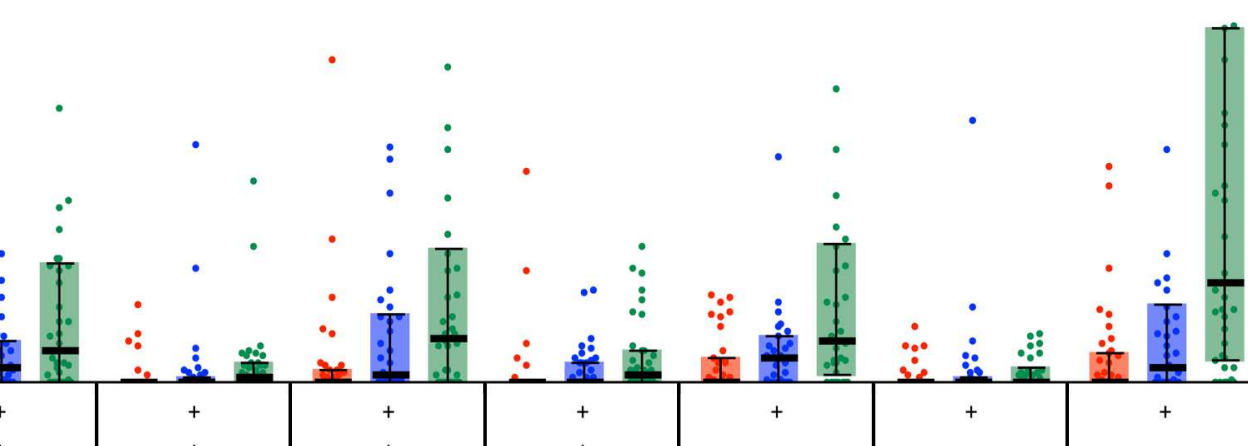

$+$

$+$

$+$

$+$

This article is protected by copyright. All rights reserved 
Figure 5 Solicited adverse events

Error bars indicate $95 \%$ confidence intervals. Fever was defined as a body temperature $\geq 38.0^{\circ} \mathrm{C}$. ${ }^{*}$ No grading of symptom severity for shivering, gastrointestinal symptoms (nausea / vomiting) and fever. N, number of participants with at least one vaccine dose administered and completed adverse events diary. Events occurred either after the first dose or second dose.

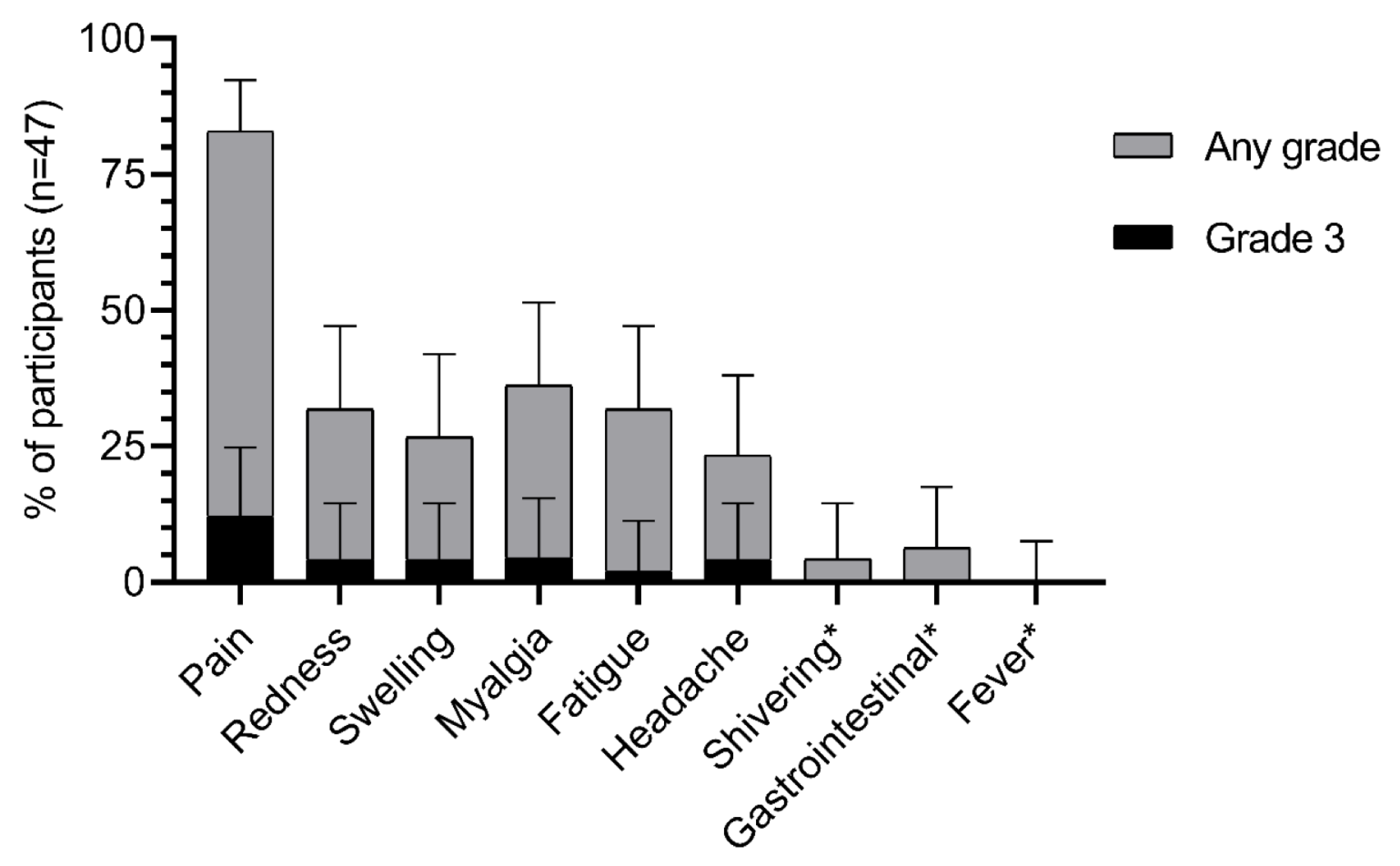

